Tôhoku Math. Journ.

28 (1976), 373-379.

\title{
ON THE NON-EXISTENCE OF FLAT CONTACT METRIC STRUCTURES
}

\author{
DAVID E. BLAIR
}

(Received March 29, 1975)

1. Introduction. It is well known that a contact manifold admits a Riemannian metric compatible with the contact structure. While such a metric is not unique, the contact structure imposes some restriction on the curvature. For example, if the characteristic vector field $\xi$ of the contact structure generates a 1-parameter group of isometries, then the sectional curvature of all plane sections containing $\xi$ is equal to 1 [1] ( $1 / 4$ in their normalization). This is a restrictive class, however as the tangent sphere bundles are usually not of this type (Tashiro [3]). Our purpose here is to show that the metric cannot in general be flat. Precisely we prove the following theorem.

THEOREM. Let $M$ be a contact manifold of dimension $\geqq 5$. Then $M$ cannot admit a contact metric structure of vanishing curvature.

The author expresses his appreciation to Professor J. Martinet for many valuable conversations.

2. Preliminaries. Let $M$ be a $(2 n+1)$-dimensional $C^{\infty}$ manifold. We say that $M$ has an almost contact structure if it admits a tensor field $\varphi$ of type $(1,1)$, a vector field $\xi$ and a 1 -form $\eta$ such that $\eta(\xi)=1$ and $\varphi^{2}=-I+\eta \otimes \xi$. From these conditions one can easily obtain $\varphi \xi=0$ and $\eta \circ \varphi=0$. Moreover on a $C^{\infty}$ manifold with an almost contact structure $(\varphi, \xi, \eta)$ there exists a Riemannian metric $g$ satisfying

$$
g(\varphi X, \varphi Y)=g(X, Y)-\eta(X) \eta(Y)
$$

for any two vector fields $X$ and $Y$ on $M$. Note that $\eta$ is the covariant form of $\xi$ and we call $(\varphi, \xi, \eta, g)$ an almost contact metric structure. We also define a 2-form $\Phi$ by $\Phi(X, Y)=g(X, \varphi Y)$.

On the other hand we say $M$ has a contact structure if it admits a global 1-form $\eta$ such that $\eta \wedge(d \eta)^{n} \neq 0$. It is well known that a manifold with a contact structure $\eta$ admits an almost contact metric structure such that

$$
d \eta(X, Y)=g(X, \varphi Y)
$$


We then say that $(\varphi, \xi, \eta, g)$ is a contact metric structure.

On a manifold with an almost contact structure $(\varphi, \xi, \eta)$, S. Sasaki and Y. Hatakeyama [2] defined four tensors $N^{(1)}, N^{(2)}, N^{(3)}$ and $N^{(4)}$ by

$$
\begin{aligned}
N^{(1)}(X, Y) & =[\varphi, \varphi](X, Y)+2 d \eta(X, Y) \xi, \\
N^{(2)}(X, Y) & =\left(\mathscr{L}_{\varphi_{X}} \eta\right)(Y)-\left(\mathscr{L}_{\varphi_{Y}} \eta\right)(X), \\
N^{(3)}(X) & =\left(\mathscr{L}_{\xi} \varphi\right) X, \\
N^{(4)}(X) & =\left(\mathscr{L}_{\xi} \eta\right)(X),
\end{aligned}
$$

where $[\varphi, \varphi]$ denotes the Nijenhuis torsion of $\varphi$ and $\mathscr{L}$ denotes Lie differentiation.

It is easy to show that for a contact metric structure $N^{(2)}$ and $N^{(4)}$ vanish [2]. Recall that the Riemannian connection $\nabla$ of $g$ is given by

$$
\begin{aligned}
2 g\left(\nabla_{X} Y, Z\right)= & X g(Y, Z)+Y g(X, Z)-Z g(X, Y) \\
& +g([X, Y], Z)+g([Z, X], Y)-g([Y, Z], X) .
\end{aligned}
$$

Using this and the coboundary formula for $d$ one can straightforwardly obtain a general formula for the covariant derivative of $\varphi$ for an almost contact metric structure $(\varphi, \xi, \eta, g)$, namely

$$
\begin{aligned}
2 g\left(\left(\nabla_{X} \varphi\right) Y, Z\right)= & 3 d \Phi(X, \varphi Y, \varphi Z)-3 d \Phi(X, Y, Z) \\
& +g\left(N^{(1)}(Y, Z), \varphi X\right)+N^{(2)}(Y, Z) \eta(X) \\
& +2 d \eta(\varphi Y, X) \eta(Z)-2 d \eta(\varphi Z, X) \eta(Y) .
\end{aligned}
$$

We close this section with the following lemma.

LeMma. On a manifold with a contact metric structure $\mathscr{L}_{\xi} \mathscr{P}$ is a symmetric operator.

Proof. Note that for a contact metric structure $(\varphi, \xi, \eta, g), \nabla_{\xi} \xi=0$ and $\nabla_{\xi} \varphi=0$. Now

$$
\begin{aligned}
g\left(\left(\mathscr{L}_{\xi} \varphi\right) X, Y\right) & =g\left(\nabla_{\xi} \varphi X-\nabla_{\varphi_{X} \xi} \xi \varphi \nabla_{\xi} X+\varphi \nabla_{X} \xi, Y\right) \\
& =g\left(-\nabla_{\varphi_{X}} \xi+\varphi \nabla_{X} \xi, Y\right)
\end{aligned}
$$

which vanishes if either $X$ or $Y$ is $\xi$. For $X$ and $Y$ orthogonal to $\xi$, $N^{(2)}=0$ becomes $\eta([\varphi X, Y])+\eta([X, \varphi Y])=0$; continuing the computation we have

$$
\begin{aligned}
g\left(\left(\mathscr{L}_{\xi} \varphi\right) X, Y\right) & =\eta\left(\nabla_{\varphi_{X}} Y\right)+\eta\left(\nabla_{X} \varphi Y\right) \\
& =\eta\left(\nabla_{Y} \varphi X\right)+\eta\left(\nabla_{\varphi_{Y}} X\right) \\
& =g\left(\left(\mathscr{L}_{\xi} \varphi\right) Y, X\right) .
\end{aligned}
$$


3. Proof of the theorem. For a contact metric structure $(\varphi, \xi, \eta, g)$, $N^{(2)}=0$ and $\Phi=d \eta$, so equation (2.1) becomes

$$
2 g\left(\left(V_{X} \varphi\right) Y, Z\right)=g\left(N^{(1)}(Y, Z), \varphi X\right)+2 d \eta(\varphi Y, X) \eta(Z)-2 d \eta(\varphi Z, X) \eta(Y) \text {. }
$$

Setting $Y=\xi$ and using the Lemma of Section 2 we obtain

$$
\begin{aligned}
-2 g\left(\varphi \nabla_{X} \xi, Z\right) & =g\left(\varphi^{2}[\xi, Z]-\varphi[\xi, \varphi Z], \varphi X\right)-2 d \eta(\varphi Z, X) \\
& =-g\left(\varphi\left(\mathscr{L}_{\xi} \varphi\right) Z, \varphi X\right)-2 g(\varphi Z, \varphi X) \\
& =-g\left(\left(\mathscr{L}_{\xi} \varphi\right) Z, X\right)-2 g(Z, X)+2 \eta(Z) \eta(X) \\
& =-g\left(\left(\mathscr{L}_{\xi} \varphi\right) X, Z\right)-2 g(X, Z)+2 g(\eta(X) \xi, Z),
\end{aligned}
$$

that is

$$
-\varphi \nabla_{X} \xi=-\frac{1}{2}\left(\mathscr{L}_{\xi} \varphi\right) X-X+\eta(X) \xi
$$

Applying $\varnothing$ we have

$$
\nabla_{X} \xi=-\frac{1}{2} \varphi\left(\mathscr{L}_{\xi} \varphi\right) X-\varphi X
$$

We denote by $R_{X Y}$ the curvature transformation $\nabla_{X} \nabla_{Y}-\nabla_{Y} \nabla_{X}-\nabla_{[X, Y]}$. Then using (3.1)

$$
\begin{aligned}
R_{\xi X} \xi & =\nabla_{\xi} \nabla_{X} \xi-\nabla_{[\xi, X]} \xi \\
& =-\frac{1}{2} \varphi \nabla_{\xi}([\xi, \varphi X]-\varphi[\xi, X])-\varphi \nabla_{\xi} X+\frac{1}{2} \varphi(\mathscr{L} \xi \varphi)[\xi, X]+\varphi[\xi, X] \\
& =\frac{1}{2} \varphi \nabla_{\xi} \nabla_{\varphi_{X}} \xi+\frac{1}{2} \nabla_{\xi} \nabla_{X} \xi-\frac{1}{2} \varphi \nabla_{\varphi[\xi, X]} \xi-\frac{1}{2} \nabla_{[\xi, X]} \xi-\varphi \nabla_{X} \xi .
\end{aligned}
$$

Therefore

$$
\frac{1}{2} R_{\xi X} \xi=\frac{1}{2} \varphi\left(\nabla_{\xi} \nabla_{\varphi_{X}} \xi-\nabla_{\left[\xi, \varphi_{X}\right]} \xi\right)+\frac{1}{2} \varphi \nabla_{\left(\mathscr{E}_{\xi} \varphi\right) X} \xi-\varphi \nabla_{X} \xi
$$

and hence since $M$ is flat

$$
\begin{aligned}
0 & =\frac{1}{2} \varphi\left(-\frac{1}{2} \varphi\left(\mathscr{L}_{\xi} \varphi\right)^{2} X-\varphi\left(\mathscr{L}_{\xi} \varphi\right) X\right)-\frac{1}{2}\left(\mathscr{L}_{\xi} \varphi\right) X-X+\eta(X) \xi \\
& =\frac{1}{4}\left(\mathscr{L}_{\xi} \varphi\right)^{2} X-X+\eta(X) \xi .
\end{aligned}
$$

Thus we define a symmetric operator $h$ by $h=(1 / 2) \mathscr{L}_{\xi} \varphi$ and we have shown that $h^{2}=-\varphi^{2}$; in particular note that $h$ has rank $2 n$. Clearly we also have $h \xi=0$, eigenvectors corresponding to non-zero eigenvalues are orthogonal to $\xi$ and the non-zero eigenvalues are \pm 1 . 
Recall that $d \eta(X, Y)=(1 / 2)\left(g\left(\nabla_{X} \xi, Y\right)-g\left(\nabla_{Y} \xi, X\right)\right)$ as can be easily deduced from the coboundary formula for $d$. Thus

$$
2 g(X, \varphi Y)=g(-\phi h X-\varphi X, Y)-g(-\varphi h Y-\varphi Y, X)
$$

giving

$$
g(\varphi h X, Y)=g(\varphi h Y, X)=-g(h Y, \varphi X)=-g(h \varphi X, Y),
$$

that is $h$ and $\varphi$ anti-commute. In particular then, if $X$ is an eigenvector of the eigenvalue $+1, \varphi X$ is an eigenvector of -1 and vice-versa. Thus the contact distribution $D$ defined by $\eta=0$ is decomposed into the orthogonal eigenspaces of \pm 1 which we denote by $[+1]$ and $[-1]$.

We now show that the distribution [-1] is integrable. If $X$ and $Y$ are vector fields belonging to $[-1],(3.1)$ gives $\nabla_{X} \xi=0, \nabla_{Y} \xi=0$. Thus since $M$ is flat $0=R_{X Y} \xi=-\nabla_{[X, Y]} \xi=\varphi h[X, Y]+\varphi[X, Y]$. But $\eta([X, Y])=$ $-2 d \eta(X, Y)=0$, thus applying $\varnothing$ we have $h[X, Y]=-[X, Y]$.

We denote by $[-1] \oplus[\xi]$ the distribution spanned by $[-1]$ and $\xi$, it is also integrable. For, any vector field belonging to [-1] can be written as $\varphi X$ for some $X \in[+1]$. Thus (3.2) becomes $0=(1 / 2) R_{\xi X} \xi=-(1 / 2) \varphi V_{[\xi, \varphi X]} \xi$ and (3.1) shows that $[\xi, \varnothing X] \in[-1]$.

Since $[-1] \oplus[\xi]$ is integrable, we can choose local coordinates $\left(u^{0}, \cdots, u^{2 n}\right)$ such that $\partial / \partial u^{0}, \cdots, \partial / \partial u^{n} \in[-1] \oplus[\xi]$ and we define local vector fields $X_{i}, i=1, \cdots, n$ by $X_{i}=\partial / \partial u^{n+i}+\sum_{j=0}^{n} f_{i}^{j} \partial / \partial u^{j}$ where the $f_{i}^{j}$ 's are functions chosen so that $X_{i} \in[+1]$. Thus $X_{1}, \cdots, X_{n}$ are $n$ linearly independent vector fields spanning [+1]. Clearly $\left[\partial / \partial u^{k}, X_{i}\right] \in[-1] \oplus[\xi]$ for $k=0, \cdots, n$ and hence $\xi$ is parallel along $\left[\partial / \partial u^{k}, X_{i}\right]$. Therefore using (3.1)

$$
0=\nabla_{\left[\partial / \partial u^{k}, X_{i}\right]} \xi=\nabla_{\partial / \partial u^{k}} \nabla_{X_{i}} \xi-\nabla_{X_{i}} \nabla_{\partial / \partial u^{k}} \xi=-2 \nabla_{\partial / \partial u^{k}} \varphi X_{\imath}
$$

from which we have that

$$
\nabla_{\varphi_{X}} \phi X_{i}=0 \text {. }
$$

Similarly, noting that $\left[X_{i}, X_{j}\right] \in[-1]$,

giving

$$
0=R_{X_{i} X_{j}} \xi=-2 \nabla_{X_{i}} \varphi X_{j}+2 \nabla_{X_{j}} \varphi X_{i}
$$

$$
\nabla_{X_{i}} \varphi X_{j}=\nabla_{X_{j}} \varphi X_{i}
$$

or equivalently

$$
\varphi\left[X_{i}, X_{j}\right]=-\left(\nabla_{X_{i}} \varphi\right) X_{j}+\left(\nabla_{X_{j}} \varphi\right) X_{i} .
$$

Using (3.3) and (3.1)

$$
0=R_{X_{i} \varphi X_{j}} \xi=-\nabla_{\left[X_{i}, \varphi X_{j}\right]} \xi=\varphi h\left[X_{i}, \varphi X_{j}\right]+\varphi\left[X_{i}, \varphi X_{j}\right]
$$


from which

$$
g\left(\left[X_{i}, \varphi X_{j}\right], X_{k}\right)=-g\left(h\left[X_{i}, \varphi X_{j}\right], X_{k}\right)=-g\left(\left[X_{i}, \varphi X_{j}\right], X_{k}\right)
$$

and hence

$$
g\left(\left[X_{i}, \varphi X_{j}\right], X_{k}\right)=0 .
$$

We now compute $\left(\nabla_{X_{i}} \varphi\right) X_{j}$ explicitly. Using (3.3), (3.6) and (3.5)

$$
\begin{aligned}
2 g\left(\left(\nabla_{X_{i}} \varphi\right) X_{j}, X_{k}\right) & =g\left([\varphi, \varphi]\left(X_{j}, X_{k}\right), \varphi X_{i}\right) \\
& =-g\left(\left[X_{j}, X_{k}\right], \varphi X_{i}\right) \\
& =g\left(-\left(\nabla_{X_{j}} \varphi\right) X_{k}+\left(\nabla_{X_{k}} \varphi\right) X_{j}, X_{i}\right) .
\end{aligned}
$$

Since $\Phi=d \eta$, the sum of the cyclic permutations of $i, j, k$ in $g\left(\left(\nabla_{X_{i}} \varphi\right) X_{j}, X_{k}\right)$ is zero. Thus our computation yields $g\left(\left(\nabla_{X_{i}} \varphi\right) X_{j}, X_{k}\right)=0$. Similarly

$$
\begin{aligned}
2 g\left(\left(\nabla_{X_{i}} \varphi\right) X_{j}, \varphi X_{k}\right) & =g\left(-\left[X_{j}, \varphi X_{k}\right]-\left[\varphi X_{j}, X_{k}\right], \varphi X_{i}\right) \\
& =g\left(-\nabla_{X_{j}} \varphi X_{k}+\nabla_{\varphi X_{k}} X_{j}-\nabla_{\varphi X_{j}} X_{k}+\nabla_{X_{k}} \varphi X_{j}, \varphi X_{i}\right)
\end{aligned}
$$

which vanishes by (3.3) and (3.4). Finally

$$
\begin{aligned}
2 g\left(\left(\nabla_{X_{i}} \varphi\right) X_{j}, \xi\right) & =g\left(\varphi^{2}\left[X_{j}, \xi\right]-\varphi\left[\varphi X_{j}, \xi\right], \varphi X_{i}\right)+2 d \eta\left(\varphi X_{j}, X_{i}\right) \\
& =2 g\left(\varphi h X_{j}, \varphi X_{i}\right)+2 g\left(X_{j}, X_{i}\right) \\
& =4 g\left(X_{j}, X_{i}\right) .
\end{aligned}
$$

Thus for any vector fields $X$ and $Y$ in $[+1]$,

$$
\left(\nabla_{X} \varphi\right) Y=2 g(X, Y) \xi \text {. }
$$

Note that (3.5) now gives $\left[X_{i}, X_{j}\right]=0$.

Before differentiating (3.7) we show that $\nabla_{X_{i}} X_{j} \in[+1]$. First note that

$$
-2 g\left(\nabla_{\varphi X_{i}} X_{j}, X_{k}\right)=2 g\left(\left(\nabla_{\varphi X_{i}} \varphi\right) X_{j}, \varphi X_{k}\right),
$$

but the right side vanishes by a computation of the type we have been doing. Therefore

$$
g\left(\nabla_{X_{i}} X_{j}, \varphi X_{k}\right)=-g\left(X_{j}, \nabla_{X_{i}} \varphi X_{k}\right)=-g\left(X_{j},\left[X_{i}, \varphi X_{k}\right]\right)=0
$$

by (3.6). That $g\left(\nabla_{X_{i}} X_{j}, \xi\right)=0$ is trivial.

Now to show the non-existence of flat contact metric structures for $\operatorname{dim} M \geqq 5$, we shall contradict the linear independence of the $X_{i}$ 's. Note also that we have so far used only the vanishing of $R_{X Y} \xi$. Equation (3.7) can be written as

$$
\nabla_{X_{i}} \varphi X_{j}-\varphi \nabla_{X_{i}} X_{j}=2 g\left(X_{i}, X_{j}\right) \xi .
$$

Differentiating this we have 


$$
\begin{aligned}
\nabla_{X_{k}} \nabla_{X_{i}} \varphi X_{j}- & \left(\nabla_{X_{k}} \varphi\right) \nabla_{X_{i}} X_{j}-\varphi \nabla_{X_{k}} \nabla_{X_{i}} X_{j} \\
& =2\left(X_{k} g\left(X_{i}, X_{j}\right)\right) \xi-4 g\left(X_{i}, X_{j}\right) \varphi X_{k} .
\end{aligned}
$$

Taking the inner product with $\varphi X_{l}$, remembering (3.7) and that $\nabla_{X_{i}} X_{j} \in$ $[+1]$, we have

$$
g\left(\nabla_{X_{k}} \nabla_{X_{i}} \varphi X_{j}, \varphi X_{l}\right)-g\left(\nabla_{X_{k}} \nabla_{X_{i}} X_{j}, X_{l}\right)=-4 g\left(X_{i}, X_{j}\right) g\left(X_{k}, X_{l}\right) \text {. }
$$

Interchanging $i$ and $k, i \neq k$ and subtracting we have

$$
0=g\left(X_{i}, X_{j}\right) g\left(X_{k}, X_{l}\right)-g\left(X_{k}, X_{j}\right) g\left(X_{i}, X_{l}\right)
$$

by virtue of the flatness and $\left[X_{i}, X_{k}\right]=0$. Setting $i=j$ and $k=l$ we have $0=g\left(X_{i}, X_{i}\right) g\left(X_{k}, X_{k}\right)-g\left(X_{i}, X_{k}\right)^{2}$ contradicting the linear independence of $X_{i}$ and $X_{k}$.

4. Remarks. In dimension 3 it is easy to construct flat contact metric structures. For example, consider $R^{3}$ with coordinates $\left(X^{1}, X^{2}, X^{3}\right)$ and define a contact structure $\eta$ by $\eta=(1 / 2)\left(\cos X^{3} d X^{1}+\sin X^{3} d X^{2}\right)$. Then $\xi$ is $2\left(\cos X^{3} \partial / \partial X^{1}+\sin X^{3} \partial / \partial X^{2}\right)$ and the metric $g$ whose components are $g_{i j}=(1 / 4) \delta_{i j}$ gives a flat contact metric structure. Geometrically we see that $\partial / \partial X^{3}$ spans the $[+1]$ distribution and $\sin X^{3} \partial / \partial X^{1}-\cos X^{3} \partial / \partial X^{2}$ spans [-1], i.e. $\xi$ is parallel along [-1] and rotates as we move parallel to the $X^{3}$-axis. Note also that $\eta$ is invariant under the group of translations generated by $\left\{X^{A} \rightarrow X^{A}+2 \pi, A=1,2,3\right\}$ and therefore the 3 dimensional torus $T^{3}$ also carries this structure. It is still an open question whether or not $T^{5}$ carries a contact structure, but if it does it can not have a flat associated metric.

Constructing the diffeomorphism of $\boldsymbol{R}^{3}$ that maps this $\eta$ to the standard contact form $\eta_{0}=(1 / 2)(d Z-Y d X)$ we see that the metric $g_{0}$ whose components are given by

$$
\frac{1}{4}\left(\begin{array}{crr}
1+Y^{2}+Z^{2} & Z & -Y \\
Z & 1 & 0 \\
-Y & 0 & 1
\end{array}\right)
$$

makes $\left(\eta_{0}, g_{0}\right)$ a flat contact metric structure.

Note that in the proof of our theorem, the vanishing of $R_{\xi X} \xi$ is enough to obtain the decomposition of the contact distribution into the \pm 1 eigenspaces of the operator $h=(1 / 2) \mathscr{L}_{\xi} \varphi$. Moreover $R_{X Y} \xi=0$ for $X$ and $Y$ in $[-1]$ is sufficient for the integrability of $[-1]$. Thus we have the following result.

THEOREM. Let $M$ be a contact manifold of dimension $2 n+1$ with contact metric structure $(\varphi, \xi, \eta, g)$. If the sectional curvature of all 
plane sections containing $\xi$ vanish, then the operator $h=(1 / 2) \mathscr{L}_{\xi} \rho$ has rank $2 n$ and the contact distribution is decomposed into \pm 1 eigenspaces of $h$. Moreover if $R_{X Y} \xi=0$ for $X, Y \in[-1], M$ admits a foliation by n-dimensional integral submanifolds of the contact distribution.

We close with an example of such a structure. Consider on $\boldsymbol{R}^{5}$ with coordinates $\left(X^{1}, \cdots, X^{5}\right)$, the standard contact structure

$$
\eta=\frac{1}{2}\left(d X^{5}-X^{3} d X^{1}-X^{4} d X^{2}\right)
$$

Then $\eta$ together with the metric $g$ whose components are given by

$$
\frac{1}{4}\left(\begin{array}{ccccc}
1+\left(X^{3}\right)^{2}+\left(X^{5}\right)^{2} & X^{3} X^{4} & X^{5} & 0 & -X^{3} \\
X^{3} X^{4} & 1+\left(X^{4}\right)^{2}+\left(X^{5}\right)^{2} & 0 & X^{5} & -X^{4} \\
X^{5} & 0 & 1 & 0 & 0 \\
0 & X^{5} & 0 & 1 & 0 \\
-X^{3} & -X^{4} & 0 & 0 & 1
\end{array}\right)
$$

is a contact metric structure. $g$ is not flat, but $R_{\xi X} \xi=0$ and $R_{\partial / \partial X^{3} \partial / \partial X^{4}} \xi=0$. Defining $h$ by $h=(1 / 2) \mathscr{L}_{\xi} \varphi$, one can easily check that $h$ determines a decomposition of the contact distribution into \pm 1 eigenspaces of $h$. [-1] is spanned by $\partial / \partial X^{3}$ and $\partial / \partial X^{4}$ and [+1] is spanned by $\partial / \partial X^{1}-X^{5} \partial / \partial X^{3}+$ $X^{3} \partial / \partial X^{5}$ and $\partial / \partial X^{2}-X^{5} \partial / \partial X^{4}+X^{4} \partial / \partial X^{5}$.

\section{REFERENCES}

[1] Y. Hatakeyama, Y. Ogawa and S. Tanno, Some properties of manifold with contact metric structures, Tôhoku Math. J., 15 (1963), 42-48.

[2] S. SASAKI and Y. HATAKEYAMA, On differentiable manifolds with certain structure which are closely related to almost contact structure II, Tôhoku Math. J., 13 (1963), 281-294.

[ 3 ] Y. TASHIRo, On contact structures of tangent sphere bundles, Tôhoku Math. J., 21 (1969), 117-143.

Institut de Recherche Mathematique Avancee

Laboratoire AsSOcié aU C.N.R.S.

7, RUE RENÉ DeSCARTES

67084 Strasbourg Cédex

AND

Michigan State University

East Lansing, Michigan 48824 
